\title{
Island morphology and adatom self-diffusion on $\operatorname{Pt}(111)$
}

\author{
Ghyslain Boisvertt and Laurent J. Lewist \\ Département de Physique et Groupe de Recherche en Physique et Technologie des Couches Minces (GCM), Université de \\ Montréal, Case Postale 6128, Succursale Centre-Ville, Montréal, Québec, Canada H3C 3J7 \\ Matthias Scheffler \\ Fritz-Haber-Institut der Max-Planck-Gesellschaft, Faradayweg 4-6, D-14195 Berlin-Dahlem, Germany
}

\section{Submitted to Physical Review B}

August 14, 2018

\begin{abstract}
The results of a density-functional-theory study of the formation energies of (100)- and (111)-faceted steps on the $\operatorname{Pt}(111)$ surface, as well as of the barrier for diffusion of an adatom on the flat surface, are presented. The step formation energies are found to be in a ratio of 0.88 in favour of the (111)-faceted step, in excellent agreement with experiment; the equilibrium shape of islands should therefore clearly be non-hexagonal. The origin of the difference between the two steps is discussed in terms of the release of stress at the surface through relaxation. For the diffusion barrier, we also find relaxation to be important, leading to a $20 \%$ decrease of its energy. The value we obtain, 0.33 $\mathrm{eV}$, however remains higher than available experimental data; possible reasons for this discrepancy are discussed. We find the ratio of step formation energies and the diffusion barrier to be the same whether using the local-density approximation or the generalized-gradient approximation for the exchange-and-correlation energy.
\end{abstract}

PACS numbers: 68.35.Bs, 68.35.Fx, 71.15.Nc

\section{INTRODUCTION}

Detailed knowledge of surface properties is important to understanding a wide variety of phenomena such as catalysis, surface reactivity, growth, etc. Of particular importance are such quantities as the step formation energy (SFE), which determines the equilibrium shape of islands on flat terraces, and the potential energy surface seen by, e.g., an adatom, which provides information on the preferred sites for adsorption and the kinetics of diffusion. We present here a detailed study of these properties for the (111) surface of platinum within the framework of density-functional theory (DFT).t

The (111) surface of fcc metals, and in particular Pt, is of interest for (at least) two reasons. First, as displayed in Fig. 1, it exhibits two possible step geometries, named according to the micro-facet that step-edge atoms form with atoms in the layer underneath, namely a (100)-faceted step, where an edge atom has a single near neighbor at the base of the step (i.e., the microfacet constitutes a square lattice) and a (111)-faceted step, where each atom has two neighbors (triangular lattice). The only difference between the two geometries, as far as nearest-neighbors are concerned, is that atoms at the base of the step have a coordination of 10 for the (100)-faceted step and 11 for the (111)-faceted step. The two steps are evidently very similar and the formation energies are thus expected to be comparable, i.e., the equilibrium island shape should be very nearly hexago- nal, with the sides consisting of, alternately, (100)- and (111)-faceted steps This has indeed been observed in the case of $\mathrm{In}^{2}$ and $\mathrm{Ag} .3 \mathrm{~F}$ For Pt, however, scanning-tunneling microscopy (STM) reveals, rather, a strong preference for (111)-faceted steps - $0.87 \pm 0.02$ as measured by the ratio of step formation energies per unit length. the theory side, DFT calculations in the local-density approximation 5 (LDA) by Stumpf and Scheffler predict this behaviour in the case of $\mathrm{Al}$, with a ratio of $\mathrm{SFE}$ of $0.93 ; 6$ this has not yet been confirmed experimentally. For Pt, in contrast, corresponding calculations by Feibelman failed to reproduce the experimental results, leading to essentially equal SFE for the two types of steps.

A second feature that makes the fcc(111) surface interesting is that it possesses two different adsorption sites: the fcc (normal) site, where an adatom sits in a position appropriate to the stacking of atomic planes in a perfect fcc crystal, and the hcp (stacking fault) site, corresponding to an hcp stacking. Both sites have three nearest neighbors, the difference between the two lying in the second-layer arrangement, as illustrated in Fig. 2. It has been shown that, for transition metals, the preferredsite for adsorption depends on the filling of the $d$ band 8.9 For Pt, DFT-LDA calculations predict the fcc site to be much more favourable, 10 by $0.12-0.18 \mathrm{eV}$, depending on the state of relaxation of the substrate. This is the largest difference obsery (so far) for late transition metals and noble metals. 111,12 Experimentally, also, there is evidence that the fcc site is preferred over the 
hcp site in $\mathrm{Pt}, 10,13$ while a precise numerical value cannot be inferred from the measurements, it jestimated that the difference should be at least $0.06 \mathrm{eV} 13$ However, despite this apparent agreement, the DFT calculations for $\operatorname{Pt}(111) 810$ have been unable to reproduce correctly the diffusion-barrier - 0.38-0.41 versus $\sim 0.26 \mathrm{eV}$ from experiment 10,14 Since it is of primary importance to have reliable and accurate energy barriers in order to predict growth (see for example Ref. 15), it is essential that this discrepancy be resolved.

Clearly, a quantitative picture of the surface properties of $\mathrm{Pt}(111)$ is still missing. In order to address this problem, we present here the results of extensive $a b$-initio total-energy calculations of the formation energies of the two kinds of steps, as well as of the energetics of adatom adsorption and diffusion. The calculations have been carried out within the LDA, but we have also carried out a series of calculations in the generalized-gradient approximation (GGA) 16 so as to assess the applicability of the LDA to this system. We find the LDA to offer a better description (compared to experiment) of the lattice constant of bulk Pt han the GGA, in agreement with previous calculations, 17,18 as well as some properties of the clean (111) surface (surface relaxation and work function). Within numerical accuracy, however, we observe no sizeable effect on the ratio of SFE and on the energetics of adatom adsorption and diffusion.

As discussed in more detail below, the electronic wavefunctions were expanded in plane waves, which enables us to deal easily, and completely, with the effect of relaxation on adsorption and adatom diffusion. In the calculations of Refs. 8 and 10, only nearest-neighbor relaxation was included, at best. We observe that proper account of atomic relaxation leads to a significant decrease, by $\sim 20 \%$, of the value of the diffusion barrier on the flat (111) surface. Our estimate for this quantity, while closer to experiment 10.14 than previous calculations, 10 however remains high -0.33 versus $\sim 0.26 \mathrm{eV}$; possible reasons for this discrepancy will be discussed. In contrast, the difference in energy between the two adsorption sites increases upon relaxing, from 0.10 to $0.17 \mathrm{eV}$.

One other important advantage of plane waves is that, contrary to the Gaussian orbitals used in Ref. 7, they are independent of the atomic positions and should therefore provide a more adequate description of the subtle difference in SFE expected here. In agreement with experiment, our highly-converged calculations indicate a clear preference for (111)-faceted steps over (100)-faceted steps, the SFE being in a ratio of 0.88 (versus about 0.87 experimentally). 1 Our calculations, further, provide a simple explanation for the origin of the energy difference between the two steps in terms of the release of surface stress through relaxation. Before discussing our results in detail, we give a brief description of our computational approach.

\section{COMPUTATIONAL DETAILS}

As already noted above, the calculations reported here were performed within the framerwork of densityfunctional theory 1 using both the LDA and the GGA 1 for the exchange-and-correlation energy. The ion cores were approximated by pseudopotentials with $5 d$ electrons treated as valence states. The pseudopotentials were generated using the semi-relativistic scheme of Troullier and Martins 19 and expressed in the Kleinman-Bytander form using the $s$ component as the local one.20 22 The electronic wave-functions were represented using a planewave basis set with kinetic energy up to $40 \mathrm{Ry}$ in the LDA and 45 Ry in the GGA. To improve convergence, the electronic states were occupied according to a Fermi distribution with $k_{B} T_{\mathrm{el}}=0.1 \mathrm{eV}$ and the total energies obtained by extrapolating to zero electronic temperature. For similar reasons, the calculations were initiated using wave-functions obtained from the self-consistent solution of the Kohn-Sham Hamiltonian in a mixed basis set composed of-pseudo-atomic orbitals and plane waves cut off at 4 Ry. 23 The minimization of the energy with respect to the electronic degrees of freedom was done using an iterative procedure.24 After achieving electronic convergence, the atoms were moved according to a damped Newton dynamics until forces became less than $0.01 \mathrm{eV} / \AA$. All the calculations were performed using the supercell approach. Details of the cell shape and size, as well as kpoint sampling, for the different geometries considered, are given along with the results in the following sections.

\section{RESULTS}

\section{A. Bulk and Surface Properties}

In order to assess the validity of our approach, and for completeness, we first determined the lattice constant of the bulk material as well as the properties of the clean $\operatorname{Pt}(111)$ surface - surface energy, relaxation, excess surface stress, and work function. As a reminder, the surface stress tensor, $g_{\alpha \beta}$, is given by

$$
g_{\alpha \beta}=\gamma \delta_{\alpha \beta}+d \gamma / d \varepsilon_{\alpha \beta}
$$

where $\gamma$ is the surface energy per unit area, $\varepsilon_{\alpha \beta}$ is the strain tensor and $\delta_{\alpha \beta}$ is the Kronecker delta function. The second term in this equation represents the excess surface stress. For the clean surface, we used a $(1 \times 1)$ supercell consisting of 5 or 7 (111) atomic planes plus $\sim 10 \AA$ of vacuum. Integration of the first Brillouin zone was done over a uniform grid of $100 \mathrm{k}$ points in the $x-y$ plane, which was found to yield well-converged results, e.g., within $2 \mathrm{meV}$ for the surface energy. The latter was calculated by comparing to a bulk-like $(1 \times 1)$ supercell containing 3 layers (and of course no vacuum). The same k-point density was used for the $x-y$ plane; to compensate for the smaller size of the cell in the $z$ direction, 
the two-dimensional grid was replicated 4 times along $z$ so as to get a density of points similar to that in the $x-y$ plane. For the excess surface stress, we varied the in-plane lattice constant for both the clean surface and the bulk, while keeping the atoms' $z$ coordinates fixed to their bulk-like values, and examined the concomitant variations in the total energy.

The results are listed in Table If, along with those from other ab-initio calculations and experimental values when available. Evidently, our LDA lattice constant is consistent with previous calculations and with experiment. The GGA value, in contrast, while in agreement with other calculations, overestimates somewhat the lattice constant, by more than $2 \%$; it is a well-known fact that the GGA yields larger lattice constant than the LDA 18 - actually overcompensates in the present case. For the surface energy and excess surface stress, our LDA results agree well with previous calculations. We note that the excess surface stress is large and positive, meaning that the Pt(111) surface is under significant tensile stress, i.e., would prefer a smaller lattice constant. Unfortunately, to our knowledge, there exists no experimental determination of these quantities. In the GGA, both the surface energy and the excess surface stress decrease. This effect of the GGA on the surface energy was actually predicted from jellium calculations. 16

We have also calculated the top and second layer relaxation, $\Delta d_{12}$ and $\Delta d_{23}$, i.e., the change in interlayer spacing relative to the bulk value. We find a small $(0.4 \%)$ outward relaxation for the top layer. This is quite a bit smaller than the value reported in Ref. 10, 1.25\%. The origin of the discrepancy between the two LDA calculations is not clear; it may be due to different choices of basis sets (LCAO in Ref. 10 versus plane waves here). The GGA, interestingly, leads to a small inward relaxation. Currently available experimental data vary widely, in the range $0-2.5 \%$, and are therefore not of much help in resolving the issue. For the second layer, theory and experiment agree that it should be insignificant, i.e., $\Delta d_{23}$ is a small fraction of a percent.

For the work function, finally, the experimental values also vary quite a bit in the range $5.77-6.10 \mathrm{eV}$. According to Kaack and Fick 40 however, the work function has a small temperature dependence, decreasing slightly with temperature. Since our calculations are performed at $0 \mathrm{~K}$, we expect that they should compare well with the largest experimental values. We find, indeed, that the LDA result, $6.07 \mathrm{eV}$, is in excellent agreement with the largest experimental number, $6.10 \mathrm{eV}$. The GGA value, in contrast, is significantly smaller - $5.70 \mathrm{eV}$ - indicating, once more, that the LDA provides a better description of Pt than the LDA. In spite of this, the two approximations will carefully be examined in the context of SFE and diffusion barriers.

\section{B. Step Formation Energy}

We come now to the heart of the matter, namely the energetics of step formation. In order to determine the SFE, we constructed vicinal surfaces (using rectangular surface cells) appropriate to each type of steps. For the (100)-faceted step, we examined both a (211) and a (332) surface; the former has 3 atoms per terrace while the latter has 5. For the (111)-faceted step, only the (221) surface, which contains 4 atoms per terrace, is considered. Again, here, a vacuum region of approximately $10 \AA$ was included in all cases.

The energies of the vicinal surfaces were determined using the same procedure as in the case of the clean surface, i.e., by comparing to an appropriate bulk model. In order to minimize the error arising from the use of different geometries, the bulk reference system for a given vicinal surface was always taken to have the same in-plane geometry as the surface. Thus, the same $\mathbf{k}$ points were used in the $x-y$ plane, while for the $z$ coordinate, the grid was adjusted to yield a comparable density. In total, the bulk supercells corresponding to the (211), (322), and (221) surfaces contained 6, 34, and 18 atoms, respectively.

The SFE is given, simply, by the difference in energy between a surface with a step and one without. Since we are dealing with vicinal surfaces here, this is equivalent to subtracting from the vicinal-surface energy (per terrace), $\sigma_{\text {vic }}$, that portion of the clean-syrface energy corresponding to the exposed (111) area. If we call $\sigma_{(111)}$ the clean-(111)-surface energy per atom and neglect stepstep interactions we find, for the (100)-faceted step:

$$
E_{(100)}^{\mathrm{SF}}=\sigma_{\mathrm{vic}}-\left(N-\frac{1}{3}\right) \sigma_{(111)}
$$

and for the (111)-faceted step:

$$
E_{(111)}^{\mathrm{SF}}=\sigma_{\mathrm{vic}}-\left(N-\frac{2}{3}\right) \sigma_{(111)}
$$

where $N$ is the number of atoms per terrace, as defined earlier.

It is evident from Eqs. 2 and 3 that, in order to determine reliably the SFE ratio, very accurate surface energies are required for both vicinal and clean surfaces. It is our purpose here to assess carefully the accuracy of our calculations through a detailed convergence study. As explained earlier, the error arising from the supercell geometry is minimized by always comparing surface and bulk energies obtained using the same in-plane periodicity and k-point density. Of course, it is essential that the energies be converged with respect to Brillouin-zone integration; for the clean (111) surface, the k-point sampling scheme used here leads to values converged within $2 \mathrm{meV}$, as discussed in Sec. IIIA. For the vicinal surfaces, we used a similar sampling scheme and, as we will see below, the error is of the order of a few $\mathrm{meV}$, so that 
differences in energy (e.g., between steps) of a few hundredths of an $\mathrm{eV}$ are significant.

The results for the (100)-faceted step under a variety of theoretical conditions are listed in Table II. First, we examine the effect of relaxation and size within the LDA. With all atoms in the bulk-like configuration (referred to as "rigid" in Table III), we find the SFE to be rather insensitive to the size of the supercell: adding two layers to the (211) slab increases the SFE by a mere $0.02 \mathrm{eV} /$ (step atom), while using a (322) surface instead of a (211) leads to a small decrease of $0.01 \mathrm{eV} /$ (step atom). (The number of layers refers to the number of (111)-like layers in the slab before a rotation is applied to make the surface vicinal.) However, upon relaxing all atoms, except those in the central (111) layer (configurations referred to as "relaxed" in Table II), the SFE is found to decrease strongly, from 0.62 to $0.43 \mathrm{eV} /$ (step atom) for the (322) surface. For the relaxed configurations, we have also examined the convergence with respect to the $\mathbf{k}$-point sampling. In all cases, the SFE changes by at most $0.01 \mathrm{eV} /$ (step atom) upon increasing the number of $\mathbf{k}$ points. Thus, we estimate the SFE for the (100)-faceted step to be $0.43 \pm 0.02$ $\mathrm{eV} /$ (step atom) within the LDA.

For the (111)-faceted step, in view of the above results, we have studied a single vicinal surface, namely the (221), at fixed and converged $\mathbf{k}$-point density, as indicated in Table III. Again, here, relaxation affects strongly the SFE, which decreases very markedly — from 0.64 to 0.38 $\mathrm{eV}$ for a 5-layer slab in the LDA. However, increasing the thickness from 5 to 7 layers brings about no significant changes in the SFE. Thus, our best LDA-SFE value for the (111)-faceted step is $0.38 \pm 0.02 \mathrm{eV} /$ (step atom).

Our calculations indicate, therefore, that the (111)faceted step has a lower formation energy than the (100)faceted step - 0.38 versus $0.43 \mathrm{eV}$ (in the LDA), leading to a ratio $E_{(111)}^{\mathrm{SF}} / E_{(100)}^{\mathrm{SF}}$ of $0.88 \pm 0.07$. This is in excellent agreement with the experimental value of $0.87 \pm 0.02$, 1 but at variance with a previous LDA calculation by Feibelman, 0 who found that the two steps are nearly equivalent, that is, $0.46 \mathrm{eV}$ for the (111)-faceted step versus $0.47 \mathrm{eV}$ for the (100), i.e., a ratio of 0.98 . The SFE values differ from Feibelman's not only in a relative sense, but also in an absolute sense: the values we find are significantly smaller, by $0.08 \mathrm{eV} /$ (step atom) for the (111)-faceted step and $0.04 \mathrm{eV} /$ (step atom) for the (100)-faceted step. Though the reasons for these differences are not clear, they may originate in the choice of basis sets: while we use plane waves, Feibelman employs Gaussian orbitals which are more sensitive to the details of the atomic configuration, as discussed in Ref. ․ In view of this, it might perhaps be the case that a Gaussian basis set lacks the accuracy needed to resolve such small energy differences as those involved here.

A value of $0.37 \mathrm{eV} /$ (step atom) for the (111)-faceted SFE has also been estimated from the experimental surface free-energy anisotropy between the (110) and (111) surfaces.41 While this corresponds quite closely to our value of $0.38 \mathrm{eV} /$ (step atom), the agreement is fortuitous since the above result was obtained assuming a value of the surface energy of $0.097 \mathrm{eV} / \AA^{2}$, much lower than that calculated here, $0.124 \mathrm{eV} / \AA^{2}$. [Using the latter value for the surface energy would lead to a (111)-faceted SFE 0.45 $\mathrm{eV} /$ (step atom) in the approach of Ref. 41.]

Recently, some concerns have been expressed regarding the procedure used here to determine the surface energy, which should diverge as the thickness of the slab increases 4243 We reinterpreted our results using the approach suggested in Ref. 43 and found only small changes in the (111) surface energy, now $0.121 \mathrm{eV} / \AA^{2}$ rather than $0.124 \mathrm{eV} / \AA^{2}$; for the SFE, we obtain now 0.41 and 0.46 $\mathrm{eV} /$ (step atom) for the (111)- and (100)-faceted step, respectively, compared to 0.38 and $0.43 \mathrm{eV} /$ (step atom) using the usual approach. The SFE ratio remains approximately unchanged, 0.89 versus 0.88 . We are thus led to conclude that, while the uncertainty on the SFE might be of the order a $0.03 \mathrm{eV}$, the value we find for the ratio is accurate to a few percent, and is not affected by the numerical procedure used.

As mentioned in the previous section, the LDA seems to provide a better description of bulk Pt, as well as of the (111) surface, than the GGA. In view of this, it is expected that the vicinal surfaces are also better represented within the LDA. The question remains open, however, because there exists no firm experimental data to compare our results to, and it is therefore of interest to calculate the SFE also within the GGA. The results are given in Tables II and III. We observe the GGA-SFE to be systematically lower than the corresponding LDA values, as is also true of the (111) surface energy. Further, convergence with respect to both size and $\mathbf{k}$-point density is similar in the two approximations. We therefore conclude to GGA values of $0.29 \pm 0.02$ and $0.25 \pm 0.02$ $\mathrm{eV} /$ (step atom) for the (100)- and (111)-faceted step, respectively. The resulting SFE ratio is $0.86 \pm 0.10$, essentially unchanged from the LDA value, namely $0.88 \pm 0.07$.

In order to understand why the two steps have different formation energies, it is of interest to consider, first, the (111)- to (100)-faceted SFE ratio in the unrelaxed (bulklike) configuration. We find, from Tables III and III, this ratio to be equal to $1.03 \pm 0.07$ (using the LDA), compared to about 0.88 for the relaxed configurations, as we have seen above. Thus, before the atoms relax, the two steps are nearly equivalent [with perhaps a slight preference for the (100)-faceted step], as could be expected from a simple nearest-neighbor model as explained in the Introduction. Evidently, therefore, the observed step anisotropy is closely related to relaxation, and this can be understood in the following way: As we have seen in Sec. III A, the $\operatorname{Pt}(111)$ surface is under large tensile stress, which can be locally relieved at steps. However, because the atomic configurations are different (albeit slightly) for the two kinds of steps, the relaxation patterns also differ, and lead to different energetics. This can in fact be seen very clearly in Fig. 3, where we plot the displacement patterns for the two types of steps: Some atoms suffer very 
large displacements — by as much as a few percent (relative to the bulk nearest-neighbor distance) for those that sit closest to the steps. More important, it is also clear from this figure that the displacements associated to the (111)-faceted step are larger than for the (100)-faceted step, i.e., the former can relieve stress more efficiently than the latter, and is thus energetically more favorable. It should be mentioned that the displacements we find here differ from Feibelman'd by as much as $1 \%$ in some cases, and might possibly explained the discrepancy between the two sets of results; this is likely related, again, to different choices of basis functions.

The relation between relaxation and stress can be understood in a more quantitative manner by considering the change in energy resulting from the displacement inwards of an edge atom, i.e., in the direction normal to the step and parallel to the terrace. Starting with both step models in their bulk-like geometry and moving an edge atom by the same amount for the two steps, we find that the (111)-faceted step, because of its triangular geometry, releases more energy than the (100)-faceted step: for a displacement of $0.14 \AA$, corresponding approximately to the observed relaxation, we find the (111)-faceted step to be already $22 \mathrm{meV}$ lower in energy than the (100)-faceted step.

Clearly, therefore, the difference in SFE arises from the large excess surface stress of the $\mathrm{Pt}(111)$ surface - 0.25 $\mathrm{eV} / \AA^{2}$ (cf. Table I), about twice as large as the surface energy, $0.124 \mathrm{eV} / \AA^{2}$. We may compare this with the corresponding situation for $\operatorname{Ir} / \operatorname{Ir}(111)$, where the equilibrium island shape is nearly hexagonal In this case, the excess surface stress is $0.128 \mathrm{eV} / \AA^{2} 25$ significantly smaller than the surface energy, $0.204 \mathrm{eV} / \AA^{2}$. Evidently, large surface energies lead to large SFE, and large excess surface stresses to large differences between the two steps. These observations therefore suggest that, as a "rule of thumb", the SFE ratio should differ from one (i.e., nonhexagonal equilibrium island shape) when the excess surface stress is sizeably larger than the surface energy. This is in fact the case of $\mathrm{Al}(111) 44$ and $\mathrm{Au}(111), 25$ while the opposite is true of Rh45 and $\mathrm{Cu} 46$ To our knowledge, no information on the equilibrium island shape is available for $\mathrm{Rh}$ and $\mathrm{Cu}$, but we would predict it to be hexagonal in both cases. For Au, reconstruction 47 is likely to be important in determining the island shape. For Al, finally, $a b$ initio calculations of the kind presented here have been performed by Stumpf and Scheffler,6 and a $\mathrm{SFE}$ ratio of 0.93 is indeed found. In this case, the ratio does not seem to be affected by relaxation, contrary to our results for $\mathrm{Pt}$, but different electronic orbitals are involved - sp for $\mathrm{Al}$ and $d$ for $\mathrm{Pt}$.

There have been other calculations of the equilibrium island shape on $\mathrm{Pt}(111)$. Using a tight-binding model, Papadia et al. found essentially no difference between the two steps in their bulk-like configuration as is the case here, but did not consider relaxation.48 Within equivalent-crystal theory, Khare and Einstein found a ratio close to unity (0.968) for the SFE, without allowing in-plane relaxation;49 also, in this approach, the surface energy is predicted to be $0.076 \mathrm{eV} / \AA^{2}$, quite a bit smaller than our $0.124 \mathrm{eV} / \AA^{2}$. Fully-relaxed calculations using the semi-empirical embedded-atom method (EAM) have also been performed and lead to a SFE ratio very close to one, 50 it is however doubtful that the EAM potential is robust enough to account for the small energy differences involved here. Other approaches have been proposed, hased on coordination- or orientation-dependent bonds, 52 which do not take relaxation effects into account.

We also list, in Tables II and III, the work function for the different surfaces examined. As expected (see for instance Ref. 53), and already observed by Feibelman, the work function is smaller for the vicinal surfaces than for the (111) surface. We also observe, in the case of the (100)-faceted step, a small dependence on the the terrace length. This agrees with Feibelman's calculations, while a stronger dependence is reported from experiment.54 This might be due to the fact that terraces studied in experiment are much wider than ours and/or surface contamination. 0

\section{Atom Adsorption and Diffusion}

We now discuss adsorption and diffusion of a Pt atom on the $\mathrm{Pt}(111)$ surface. For these calculations, an adatom is added on one surface of the $\mathrm{Pt}$ slab while the other surface is constrained to its bulk-like configuration. In order to determine the energies at the two adsorption sites as well as the barrier for diffusion, we considered both a $(2 \times 2)$ and a $(3 \times 3)$ cell with, again, approximately $10 \AA$ of vacuum. Unless otherwise noted, the integration over reciprocal space was performed using a mesh of 16 equidistant $\mathbf{k}$ points for the $(2 \times 2)$ cell and $9 \mathbf{k}$ points for the $(3 \times 3)$ cell.

First, starting with the $(2 \times 2)$ system, we examined convergence with respect to the number of layers, which we varied from 3 to 6 . The results are given in Table IV. We observe significant changes upon going from 3 to 4 (for a given state of relaxation), while increasing this number further brings about changes of at most $0.01 \mathrm{eV}$; thus, 4 layers seem to be sufficient for reliable estimates of both the diffusion barrier, $E_{\mathrm{d}}$, and the difference in adsorption energies, $\Delta E_{a d s}$.

The barrier we obtain for the unrelaxed substrate, 0.41 $\mathrm{eV}$, and the difference in adsorption energies, $0.10 \mathrm{eV}$, agree well with previous LDA calculations. 10 It is however clear from Table IV that relaxation effects are again here important: If we allow the topmost layer to relax, $E_{\mathrm{d}}$ decreases to $0.34 \mathrm{eV}$ and $\Delta E_{\text {ads }}$ increases to $0.17 \mathrm{eV}$; including second-layer relaxation as well results in relatively minor changes to the energies (less than $0.02 \mathrm{eV}$ ).

It should be noted that, because of the asymmetry between the two adsorption sites, there are in fact two barriers for diffusion. However, the difference between 
the two barriers is such that, for temperatures of interest, it is the highest-energy barrier that limits diffusion, i.e., the adatom will escape rapidly from the low-energy adsorption state but get trapped in the high-energy site.

We have also examined convergence with respect to k-point sampling, energy cutoff, and lateral size. As indicated in Table IV, we find in all cases very modest changes of at most $0.01 \mathrm{eV}$. Likewise, using the GGA does not lead to appreciable changes to the energy barrier, while $\Delta E_{\text {ads }}$ decreases by about $0.03 \mathrm{eV}$. For the reasons discussed in Sec. III A, we suspect that the LDA values are more accurate; our best, highly-converged estimates of $E_{\mathrm{d}}$ and $\Delta E_{\text {ads }}$ are thus $0.33 \pm 0.03$ and $0.17 \pm 0.03$ $\mathrm{eV}$, respectively. As discussed in Ref. 8, the large difference between the fcc and the hcp site is related to the angular character of the $d$ orbitals, whose bonding strength depends on the filling and radial quantum number of the bands. Due to this difference, the transition site for jump diffusion does not lie exactly midway between the two equilibrium sites but, rather, about $0.07 \AA$ towards the hcp site. The potential energy surface is however very flat in this region, changing by no more than $0.01 \mathrm{eV}$ upon going from the transition to the midpoint site.

There is very little experimental information available for $\Delta E_{\text {ads }}$; as mentioned in the Introduction, only a lower limit of $0.06 \mathrm{eV}$ has been determined,13 and this is consistent with our results. For the diffusion barrier, a value of $0.25 \pm 0.02 \mathrm{eV}$ has been inferred from field-ion microscopy (FIM) measurements in the temperature range 92-100 K.1 Also, based on a comparison between STM measurements of the island density and kinetic Monte Carlo simulations between $110 \mathrm{~K}$ and $160 \mathrm{~K}$, an estimate of $0.26 \pm 0.01 \mathrm{eV}$ is obtained.14 Both values agree, but disagree with our result of $0.33 \pm 0.03 \mathrm{eV}$, which is surprising in view of the high-level of accuracy and convergence of our calculations.

One possible explanation for this disagreement would be the neglect, in our calculations, of dynamical effects. These cannot be assessed directly from first-principles but have been shown to be insignificant at low temperatures, either from the calculation of dynamical corrections to the transition state thepry 55 or from empirical molecular-dynamics simulatipns.56 58 Experimentally, it is clear that the FIM value 10 suffers from poor statistics - only two points are used to determine the Arrhenius parameters - and therefore the error bar is large. Concerning the STM experiment, it has been pointed out by the authors that small-cluster mobility could affect the Monte-Carlo estimate of the barrier if its energy is close to that for adatom diffusion, in which case the quoted value would be a lower bound to the actual barrier. As a final point, it should be mentioned that many different empirical potentials have been used to determine the diffusion barrier of $\mathrm{Pt}$ adatoms on $\mathrm{Pt}(111) 10.5963$ yielding to values in the range $0.01-0.18 \mathrm{eV}$, i.e., much lower than the experimental value, which we argue, is a lower bound to the actual barrier. Thus, such models are clearly too crude to provide a proper description of the energetics of diffusion for the present system.

\section{SUMMARY}

We have used highly-accurate ab-initio methods to calculate the ratio of (111)- to (100)-faceted step formation energies on the (111) surface of Pt. We find, in excellent agreement with experiment, (111)-faceted steps to be favoured over (100) in a ratio of about 0.88 ; as a consequence, the island on this surface should be clearly non-hexagonal. The difference between the two steps is related to the large tensile stress of the $\mathrm{Pt}(111)$ surface, which is released in a different manner because of differences in the local topology. Our calculations underline the importance of relaxation in such cases: while the two steps are about equivalent for the unrelaxed substrate, relaxation does bring about large changes in the formation energies. Likewise, relaxation is important to a proper determination of equilibrium-site energies on the flat (111) surface. We find the fcc site to be preferred over the hcp by a sizeable $0.17 \mathrm{eV}$ (after full relaxation), consistent with experiment which provides a lower bound of $0.06 \mathrm{eV}$ for the difference between the two sites.

We have also calculated the energy barrier for adatom diffusion and found a fully-relaxed value of $0.33 \mathrm{eV}$. While constituting an improvement over previous calculations, this value remains larger than experiment, by about 0.07 $\mathrm{eV}$. The discrepancy might be due to limitations of our theoretical approach (e.g., finite size), but it might also due to errors in the interpretation of the experimental data - poor statistics, neglect of small-cluster contributions (e.g., dimers) to mass transport, i.e., incorrect assumption regarding the critical nucleus size. More experiments are needed to clarify this point. Likewise, it would be of interest that measurements of the SFE and difference in energy between fcc and hcp sites be carried out so as to assess the validity of LDA (versus GGA) in the present context.

\section{ACKNOWLEDGMENTS}

G.B. acknowledges the warm hospitality of the Theory Department of the Fritz-Haber-Institut where a large part of this work was performed. We are grateful to Martin Fuchs for help with generating the pseudopotentials and to Alexander Kley, Christian Ratsch, Paolo Ruggerone, Ari P Seitsonen, and Byung Deok Yu for stimulating discussions. This work was supported by grants from the Natural Sciences and Engineering Research Council (NSERC) of Canada and the "Fonds pour la formation de chercheurs et l'aide à la recherche" (FCAR) of the Province of Québec. One of us (G.B.) is thankful to NSERC and FCAR for financial support. We are grateful to the "Services informatiques de l'Université de Montréal" for generous allocations of computer resources. 
Part of the calculations reported here were carried out on the IBM/SP-2 at the CACPUS ("Centre d'applications du calcul parallèle de l'Université de Sherbrooke").

* Electronic mail address: boisver@physcn.umontreal.ca

$\dagger$ To whom correspondence should be addressed; e-mail address: lewis@physcn.umontreal.ca

${ }^{1}$ P. Hohenberg and W. Kohn, Phys. Rev. 136, B864 (1964); W. Kohn and L.J. Sham, Phys. Rev. 140, A1133 (1965).

${ }^{2}$ T.-Y. Fu, Y.-R. Tzeng, and T.T. Tsong, Surf. Sci. Lett. 366, L691 (1996).

${ }^{3}$ K. Morgenstern, G. Rosenfeld, and G. Comsa, Phys. Rev. Lett. 76, 2113 (1996).

${ }^{4}$ T. Michely and G. Comsa, Surf. Sci. 256, 217 (1991); T. Michely, M. Hohage, M. Bott, and G. Comsa, Phys. Rev. Lett. 70, 3943 (1993).

${ }^{5}$ D.M. Ceperley and B.J. Alder, Phys. Rev. Lett. 45, 566 (1980); J. Perdew and A. Zunger, Phys. Rev. B 23, 5048 (1981).

${ }^{6}$ R. Stumpf and M. Scheffler, Phys. Rev. B 53, 4958 (1996).

${ }^{7}$ P.J. Feibelman, Phys. Rev. B 52, 16845 (1995).

8 J.J. Mortensen, B. Hammer, O.H. Nielsen, K.W. Jacobsen, and J.K. Nørskov, Springer Series on Solid State Physics, Ed. A. Okiji, 121, 173 (1996).

${ }^{9}$ B. Piveteau, D. Spanjaard, and M.C. Desjonquères, Surf. Sci. 287/288, 946 (1993).

${ }^{10}$ P.J. Feibelman, J.S. Nelson, and G.L. Kellogg, Phys. Rev. B 49, 10548 (1994)

${ }^{11}$ G. Boisvert, L.J. Lewis, M.J. Puska, and R.M. Nieminen, Phys. Rev. B 52, 9078 (1995).

12 S.C. Wang and G. Ehrlich, Phys. Rev. Lett. 68, 1160 (1992).

13 A. Gölzhäuser and G. Ehrlich, Phys. Rev. Lett. 77, 1334 (1996).

14 M. Bott, M. Hohage, M. Morgenstern, T. Michely, and G. Comsa, Phys. Rev. Lett. 76, 1304 (1996).

${ }^{15}$ P. Ruggerone, C. Ratsch, and M. Scheffler, to be published in Growth and Properties of Ultrathin Epitaxial Layers, edited by D.A. King and D.P. Woodruff (Elsevier Science, Amsterdam, 1997).

16 J.P. Perdew, J.A. Chevary, S.H. Vosko, K.A. Jackson, M.R. Pederson, D.J. Singh, and C. Fiolhais, Phys. Rev. B 46, 6671 (1992).

${ }^{17}$ V. Ozoliņš and M. Körling, Phys. Rev. B 48, 18304 (1993).

18 A. Khein, D.J. Singh, and C.J. Umrigar, Phys. Rev. B 51, 4105 (1995).

19 N. Troullier and J.L. Martins, Solid State Commun. 74, 613 (1990); Phys. Rev. B 43, 1993 (1991).

${ }^{20}$ L. Kleinman and D.M. Bylander, Phys. Rev. Lett. 48, 1425 (1982).

${ }^{21}$ X. Gonze, R. Stumpf, and M. Scheffler, Phys. Rev. B 44, 8503 (1991).

${ }^{22}$ M. Fuchs et al., unpublished.

${ }^{23}$ M. Bockstedte, A. Kley, J. Neugebauer, and M. Scheffler, submitted to Computer Phys. Commun.
${ }^{24}$ R. Stumpf and M. Scheffler, Computer Phys. Commun. 79, 447 (1994).

${ }^{25}$ R.J. Needs and M. Mansfield, J. Phys.: Condens. Matter 1, 7555 (1989); M.C. Payne, N. Roberts, R.J. Needs, M. Needels, and J.D. Joannopoulos, Surf. Sci. 211/212, 1 (1989).

${ }^{26}$ P.J. Feibelman, Phys. Rev. B 51, 17867 (1995).

27 Y.S. Touloukian, R.K. Kirby, R.E. Taylor, and P.D. Desai, Thermophysical Properties of Matter, (IFI/Plenum, New York, 1977), Vol. 12.

${ }^{28}$ L.L. Kesmodel, C.P. Stair, and G.A. Somorjai, Surf. Sci. 64, 342 (1977).

${ }^{29}$ K. Hayek, H. Glassl, A. Gutmann, H. Leonhard, M. Prutton, S.P. Tear, and M.R. Welton-Cook, Surf. Sci. 152/153, 419 (1985).

${ }^{30}$ E. Bøgh and I. Stensgaard, Phys. Lett. A 65, 357 (1978).

31 J.F. van der Veen, R.C. Smeenk, R.M. Tromp, and F.W. Saris, Surf. Sci. 79, 219 (1979).

${ }^{32}$ N. Materer, U. Starke, A. Barbieri, R. Döll, K. Heinz, M.A. van Hove, and G.A. Somorjai, Surf. Sci. 325, 207 (1995).

33 D.L. Adams, H.B. Nielsen, and M.A. van Hove, Phys. Rev. B 20, 4789 (1979).

34 J.A. Davies, D.P. Jackson, P.R. Norton, D.E. Posner, and W.N. Unertl, Solid State Commun. 34, 41 (1980).

${ }^{35}$ M. Kiskinova, G. Pirug, and H.P. Bonzel, Surf. Sci. 133, 321 (1983).

${ }^{36}$ M. Salmerón, S. Ferrer, M. Jazzar, and G.A. Somorjai, Phys. Rev. B 28, 6758 (1983).

37 G.N. Derry and Z. Ji-Zhang, Phys. Rev. B 39, 1940 (1989).

${ }^{38}$ M. Alnot, I.J. Ehrhardt, and J.A. Barnard, Surf. Sci. 208, 285 (1989).

39 A. Cassuto, M. Mane, M. Hugenschmidt, P. Dolle, and J. Jupille, Surf. Sci. 237, 63 (1990).

${ }^{40}$ M. Kaack and D. Fick, Surf. Sci. 342, 111 (1995).

${ }^{41}$ H.P. Bonzel, Surf. Sci. 328, L571 (1995).

42 J.C. Boettger, Phys. Rev. B 49, 16798 (1994).

${ }^{43}$ V. Fiorentini and M. Methfessel, J. Phys. Condens. Matter 8, 6525 (1996).

${ }^{44}$ R.J. Needs, Phys. Rev. Lett. 58, 53 (1987).

${ }^{45}$ A. Filippetti, V. Fiorentini, K. Stokbro, R. Valente, and S. Baroni, in Materials Theory, Simulations, Parallel Algorithms, edited by E. Kaxiras and J.D. Joannopoulos, MRS Proceedings 408, (Materials Research Society, Pittsburgh, 1996).

${ }^{46}$ G. Boisvert and L.J. Lewis, unpublished.

47 J.V. Barth, H. Brune, G. Ertl, and R.J. Behm, Phys. Rev. B 42, 9307 (1990); A.R. Sandy, S.G.J. Mochrie, D.M. Zehner, K.G. Huang, and D. Gibbs, Phys. Rev. B 43, 4667 (1991).

${ }^{48}$ S. Papadia, M.C. Desjonquères, and D. Spanjaard, Phys. Rev. B 53, 4083 (1996).

49 S.V. Khare and T.L. Einstein, Surf. Sci. 314, L857 (1994).

${ }^{50}$ R.C. Nelson, T.L. Einstein, S.V. Khare, and P.J. Rous, Surf. Sci. 295, 462 (1993).

${ }^{51}$ M.C. Fallis and C.Y. Fong, Phys. Rev. B 54, 10869 (1996).

${ }^{52}$ G.T. Barkema, M.E.J. Newman, and M. Breeman, Phys. Rev. B 50, 7946 (1994).

${ }^{53}$ M. Methfessel, D. Hennig, and M. Scheffler, Phys. Rev. B 46, 4816 (1992).

${ }^{54}$ K. Besocke, B. Krahl-Urban, and H. Wagner, Surf. Sci. 68, 
39 (1977).

${ }^{55}$ J.M. Cohen and A.F. Voter, J. Chem. Phys. 91, 5082 (1989).

${ }^{56}$ G. Boisvert and L.J. Lewis, Phys. Rev. B 54, 2880 (1996).

${ }^{57}$ R. Ferrando and G. Tréglia, Surf. Sci. 331-333, 920 (1995).

${ }^{58}$ G.C. Kallinteris, G.A. Evangelakis, and N.I. Papanicolaou, Surf. Sci. 369, 185 (1996).

${ }^{59}$ J. Majerus, N.J. Castellani, and P. Légaré, Surf. Sci. 311, L661 (1994).

${ }^{60}$ Y. Li and A.E. DePristo, Surf. Sci. 351, 189 (1996).

${ }^{61}$ C.L. Liu, J.M. Cohen, J.B. Adams, and A.F. Voter, Surf. Sci. 253, 334 (1991).

${ }^{62}$ P. Stoltze, J. Phys. Condens. Matter 6, 9495 (1994).

${ }^{63}$ D.W. Bassett and P.R. Webber, Surf. Sci. 70, 520 (1978). 
TABLE I. Bulk and clean (111) surface properties of platinum: lattice constant $a$, surface energy $\sigma$, excess surface stress $\tau$, top and second layer relaxation, $\Delta d_{12}$ and $\Delta d_{23}$, and work function $W$.

\begin{tabular}{|c|c|c|c|c|c|c|}
\hline & $\begin{array}{c}a \\
(\AA)\end{array}$ & $\begin{array}{c}\sigma \\
\left(\mathrm{eV} / \AA^{2}\right)\end{array}$ & $\begin{array}{c}\tau \\
\left(\mathrm{eV} / \AA^{2}\right)\end{array}$ & $\begin{array}{c}\Delta d_{12} \\
\left(\% d_{\text {bulk }}\right)\end{array}$ & $\begin{array}{c}\Delta d_{23} \\
\left(\% d_{\text {bulk }}\right)\end{array}$ & $\begin{array}{c}W \\
(\mathrm{eV})\end{array}$ \\
\hline$\overline{\text { Present LDA }}$ & 3.92 & 0.124 & 0.25 & 0.4 & -0.2 & 6.07 \\
\hline Present GGA & 口 4өीमа п & $0.097 \square$ & ק.22 & -04 & $0.0 \square$ & $5.7 \beta_{1}$ \\
\hline Other LDA & $3.87,253.89$, $10,1,1,263.90$ & $0.13725,26$ & $0.213,250.28926$ & 1.2510 & $-0.05 \mathrm{td}$ & $6.10^{10}$ \\
\hline $\begin{array}{l}\text { Other GGA } \\
\text { expt. }\end{array}$ & 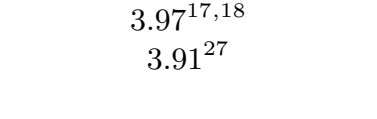 & & & 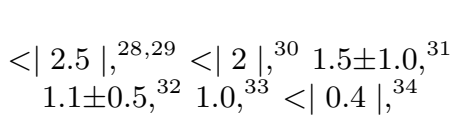 & 0.032 & $5.77-6.10354$ \\
\hline
\end{tabular}

TABLE II. Step formation energy (SFE) for the (100)-faceted step on $\mathrm{Pt}(111)$ (in eV per step atom) and work function $W$ for the corresponding vicinal surfaces (see text), as a function of the number of (111) layers, $N_{L}$, number of k points, $N_{k}$, and approximation scheme for the exchange-and-correlation energy $(\mathrm{XC})$.

\begin{tabular}{|c|c|c|c|c|c|}
\hline surface & $N_{L}$ & $N_{k}$ & $\mathrm{XC}$ & $\begin{array}{c}\mathrm{SFE} \\
(\mathrm{eV} / \mathrm{at})\end{array}$ & $\begin{array}{c}W \\
(\mathrm{eV}) \\
\end{array}$ \\
\hline (211)-rigid & 5 & 8 & LDA & 0.63 & 5.88 \\
\hline (211)-relaxed & 5 & 8 & LDA & 0.45 & 5.92 \\
\hline (211)-relaxed & 5 & 12 & LDA & 0.46 & 5.93 \\
\hline (211)-rigid & 7 & 8 & LDA & 0.65 & 5.89 \\
\hline (211)-relaxed & 7 & 8 & LDA & 0.47 & 5.94 \\
\hline (211)-rigid & 5 & 8 & GGA & 0.50 & 5.58 \\
\hline (211)-relaxed & 5 & 8 & GGA & 0.34 & 5.63 \\
\hline (211)-relaxed & 5 & 12 & GGA & 0.34 & 5.64 \\
\hline (211)-rigid & 7 & 8 & GGA & 0.52 & 5.59 \\
\hline (211)-relaxed & 7 & 8 & GGA & 0.35 & 5.63 \\
\hline (322)-relaxed & 5 & 4 & LDA & 0.44 & 5.96 \\
\hline (322)-rigid & 5 & 8 & LDA & 0.62 & 5.94 \\
\hline (322)-relaxed & 5 & 8 & LDA & 0.43 & 5.95 \\
\hline (322)-rigid & 5 & 4 & GGA & 0.46 & 5.64 \\
\hline (322)-relaxed & 5 & 4 & GGA & 0.29 & 5.66 \\
\hline
\end{tabular}

TABLE III. Same as Table II but for the (111)-faceted step.

\begin{tabular}{lcccr}
\hline \hline surface & $N_{L}$ & $N_{k}$ & XC & $\begin{array}{c}\text { SFE } \\
(\mathrm{eV} / \mathrm{at})\end{array}$ \\
\hline $\begin{array}{l}221) \text {-rigid } \\
\text { (221)-relaxed }\end{array}$ & 5 & 8 & LDA & 0.64 \\
$(221)$-relaxed & 5 & 8 & LDA & 0.38 \\
$(\mathrm{eV})$
\end{tabular}


TABLE IV. Adatom diffusion barrier $E_{\mathrm{d}}$ and difference in adsorption energies between fcc and hcp sites, $\Delta E_{\mathrm{ads}}=E_{\mathrm{ads}}^{\mathrm{fcc}}-E_{\mathrm{ads}}^{\mathrm{hcp}}$, for $\mathrm{Pt}$ on $\mathrm{Pt}(111)$ under various calculational conditions, as discussed in the text.

\begin{tabular}{|c|c|c|c|c|c|}
\hline$\overline{\mathrm{XC}}$ & supercell & allowed to relax & $\mathbf{k}$ point grid & $\begin{array}{c}E_{\mathrm{d}} \\
(\mathrm{eV})\end{array}$ & $\begin{array}{c}\Delta E_{\mathrm{ads}} \\
(\mathrm{eV})\end{array}$ \\
\hline$\overline{\mathrm{LDA}}$ & $(2 \times 2), 3$-layer & adatom only & $4 \times 4$ & 0.47 & 0.22 \\
\hline $\mathrm{LDA}$ & $(2 \times 2), 3$-layer & adatom + top layer & $4 \times 4$ & 0.41 & 0.26 \\
\hline LDA & $(2 \times 2)$, 4-layer & adatom only & $4 \times 4$ & 0.41 & 0.10 \\
\hline LDA & $(2 \times 2)$, 4-layer & adatom + top layer & $4 \times 4$ & 0.34 & 0.17 \\
\hline GGA & $(2 \times 2), 4$-layer & adatom + top layer & $4 \times 4$ & 0.33 & 0.14 \\
\hline LDA & $(2 \times 2), 4$-layer & adatom +2 top layers & $4 \times 4$ & 0.36 & 0.17 \\
\hline LDA & $(2 \times 2)$, 4-layer & adatom + top layer & $5 \times 5$ & 0.35 & 0.17 \\
\hline $\operatorname{LDA}(50 \mathrm{Ry})$ & $(2 \times 2)$, 4-layer & adatom + top layer & $4 \times 4$ & 0.35 & \\
\hline LDA & $(2 \times 2)$, 5-layer & adatom + top layer & $4 \times 4$ & 0.33 & \\
\hline LDA & $(2 \times 2), 6$-layer & adatom + top layer & $4 \times 4$ & 0.35 & \\
\hline LDA & $(3 \times 3)$, 4-layer & adatom + top layer & $3 \times 3$ & 0.33 & \\
\hline
\end{tabular}




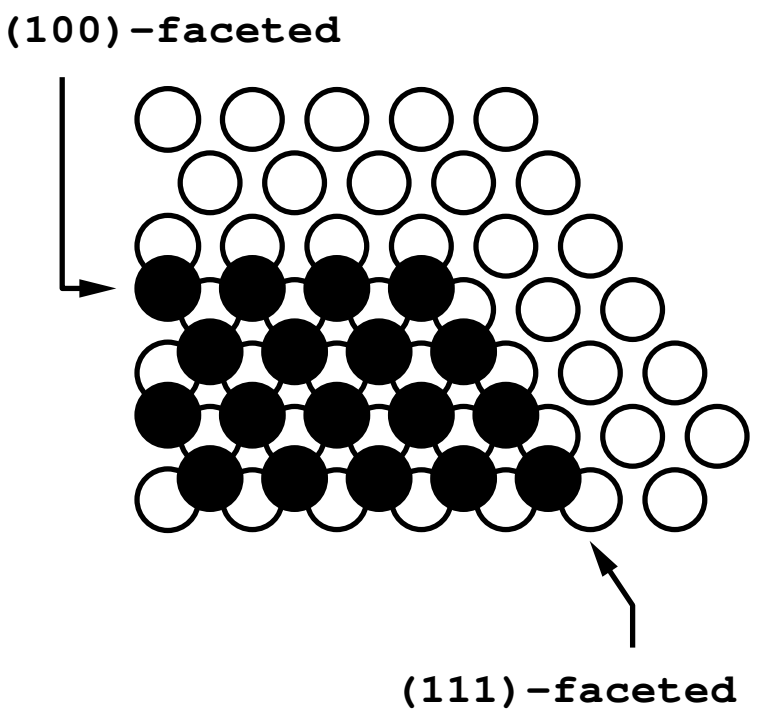

FIG. 1. Top view of an island (filled circles) on a fcc(111) surface indicating the two possible step geometries.

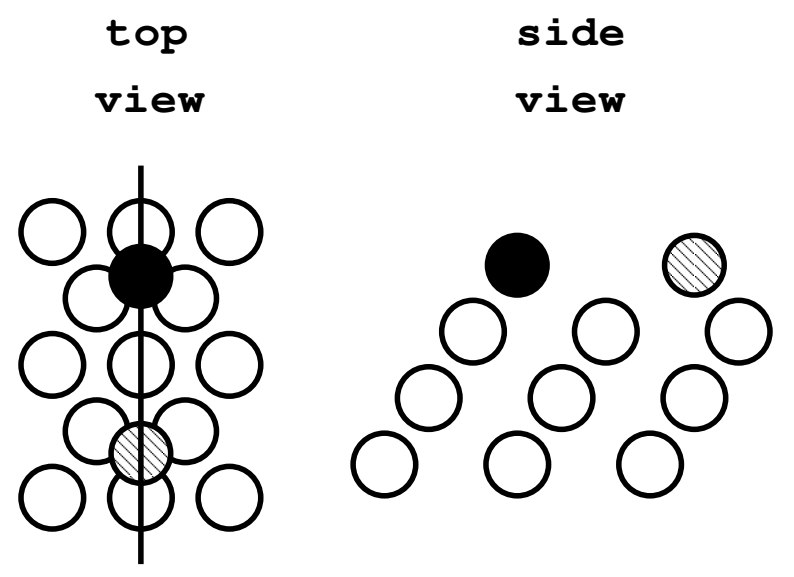

FIG. 2. Top and side view of the two different adsorption sites on a fcc (111) surface: fcc site (filled circle) and hcp site (dashed circle). The side view corresponds to the plane indicated by the straight line in the top view. (a) (100)-faceted step

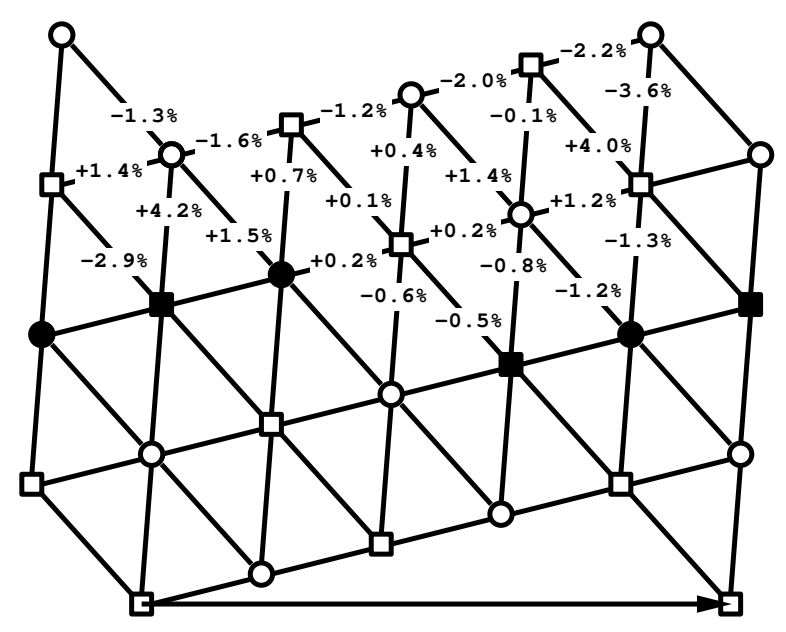

(b) (111)-faceted step

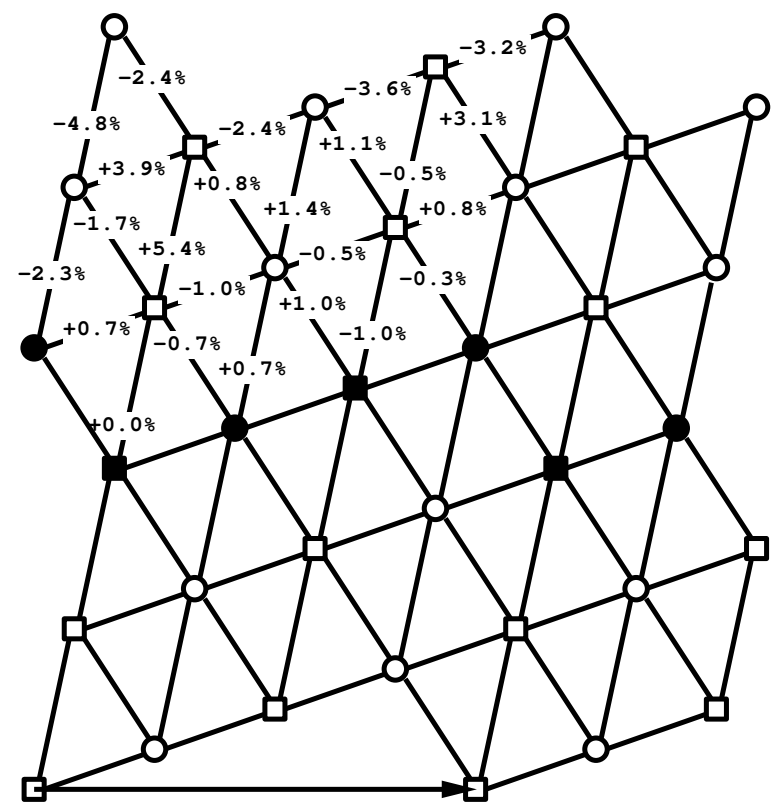

FIG. 3. Side view of the supercell showing the relaxed geometry for (a) the (100)-faceted step [as obtained from the (322) surface] and (b) the (111)-faceted step [from the (221) surface]. The filled symbols represent the middle (111) layer, which is fixed in its bulk-like position. The circles are in the plane of the sheet while the squares are in a different plane, distant by $d / 2$, where $d$ is the nearest-neighbor distance. The arrows indicate the periodicity of the supercell. 